\title{
Layer Reduction in Driven 2D-Colloidal Systems through Microchannels
}

\author{
M. Köppl, P. Henseler, A. Erbe, ${ }^{*}$ P. Nielaba, and P. Leiderer \\ Fachbereich für Physik, Universität Konstanz, 78457 Konstanz, Germany
}

(Received 9 June 2006; published 14 November 2006)

\begin{abstract}
The transport behavior of a system of gravitationally driven superparamagnetic colloidal particles is investigated. The motion of the particles through a narrow channel is governed by magnetic dipole interactions, and a layered structure forms parallel to the walls. The arrangement of the particles is perturbed by diffusion and the motion induced by gravity leading to a density gradient along the channel. Our main result is the reduction of the number of layers. Experiments and Brownian dynamics simulations show that this occurs due to the density gradient along the channel.
\end{abstract}

DOI: 10.1103/PhysRevLett.97.208302

Lattice defects can be introduced in perfectly ordered crystals by deformations due to external forces. Despite their importance, the dynamical behavior of single dislocations is still rather poorly understood, because they are difficult to generate and observe on the atomic scale. Therefore, the observation of this behavior in a model system will lead to insights that can be transferred to a broad range of systems. Studies of isolated lattice defects have recently become possible in static systems of colloidal crystals [1]. Experiments on the behavior of such defects in a nonequilibrium system can lead to a better understanding of the transport behavior of a wide range of systems.

In biological systems, the transport of interacting particles through narrow constrictions is of high importance for many processes, for example, for the size selectivity of transport in ion channels [2]. The complexity of such systems allows one to only make hypotheses on the underlying physics governing such phenomena. Model systems that can be easily accessed experimentally can reveal many of the underlying processes [3]. This requires studies of particle transport through channels with variously shaped walls. In order to perform these studies, transport through channels with straight walls has to be well understood in experiments shown in this Letter.

In this work, we report on studies of the transport behavior of colloids in a quasi-two-dimensional (2D) setup. The colloids are superparamagnetic; therefore, the interaction energy can be continuously tuned by the application of an external magnetic field [4]. The particles are driven by gravity through a narrow constriction (channel). Such driven diffusive systems serve as model systems for theoretical studies of nonequilibrium behavior [5]. In addition, such a system resembles the classical case of a quantum point contact in mesoscopic electronics [6,7] or in metallic single atom contacts [8,9]. These contacts exhibit transport in electronic channels due to quantization effects. These quantum channels can be seen as analogous to the layers in the macroscopic transport, since both occur due to the interaction of the particles with the confining potential. A classical version of a similar scenario can be
PACS numbers: 82.70.Dd, 61.72.-y, 64.60.Cn, 75.40.Mg

built on a liquid helium surface, which is loaded with charges. For such a system, the formation of layers has been reported as well [10]. The change of the number of such layers in the vicinity of a constriction has been predicted from Langevin dynamics simulations of Yukawa particles [11]. The main advantage of the use of superparamagnetic colloids instead of electrons is given by the size of the colloids, which can be easily monitored in a standard video microscopy setup. All relevant parameters can be gathered from the configuration data.

We compare the experimental results gained from video microscopy with Brownian dynamics (BD) simulations of particle flow through constrictions under very similar conditions. The results of both cases show agreement in the essential features. The BD simulations can, therefore, be

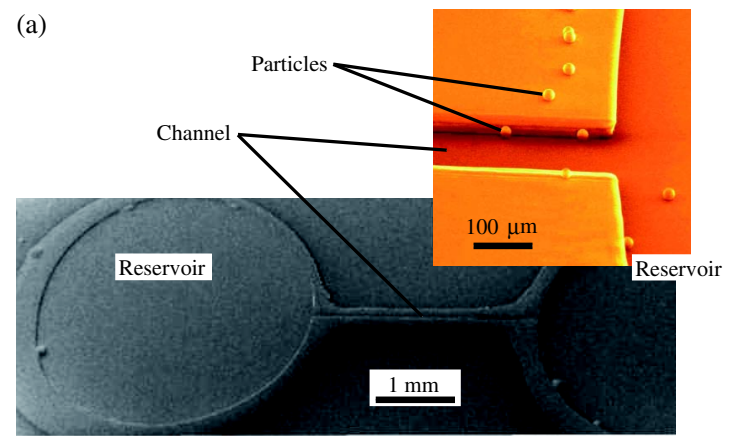

(b)

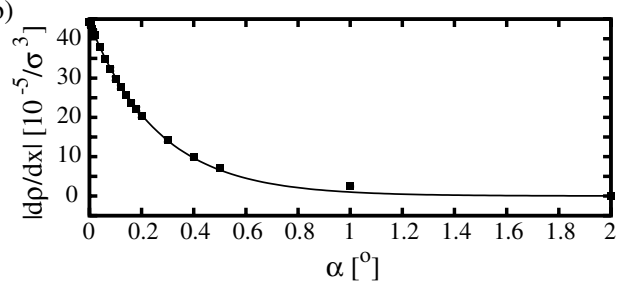

FIG. 1 (color online). (a) SEM pictures of the channel: Shown are an overview of the channel and a zoom to the region of the channel entrance. Some dried particles inside and outside of the channel can be seen as well. (b) Simulation results of the linear fits to the local density (neglecting the first and last $10 \%$ of the channel) in dependence of the inclination $\alpha$. 
used to test the robustness of the effects for a much wider range of possible configurations than experimentally possible. The main focus of this discussion will be on the phenomenon of layer reduction. Layering of particles in 2D systems due to the presence of walls is well known for equilibrium systems [12,13].

Scanning electron microscopy (SEM) pictures of the channel setup and of the channel entrance together with some dried particles inside and outside of the channel can be seen in Fig. 1(a). Two particle reservoirs and a connecting channel are defined on a lower substrate using UV lithography. The channel (60 $\mu \mathrm{m}$ wide and $2 \mathrm{~mm}$ long) is filled with a suspension of superparamagnetic particles, which are commercially available (Dynal, particle diameters $\sigma=4.55 \mu \mathrm{m}$, suspended in water). Gravity confines the colloidal particles to the surface of the channel due to the density mismatch between the particles and the liquid. So the system is quasi-2D, as long as the magnetic interactions do not lead to out-of-plane motion of the particles. Thus, for an applied uniform magnetic field perpendicular to the monolayer, the particle interaction is purely repulsive, and its strength at distance $r_{i j}$ is given by $V_{i j}\left(r_{i j}\right)=$ $\left(\mu_{0} / 4 \pi\right) M^{2} / r_{i j}^{3}$, with the magnetic dipole moments $M=$ $\chi_{\mathrm{eff}} B$ of the particles. The importance of the pair interaction can be characterized by the dimensionless interaction strength $\Gamma=\mu_{0} M^{2}(\pi \rho)^{3 / 2} /\left(4 \pi k_{B} T\right)$, where $\rho$ denotes the number density of the particles, $k_{B}$ the Boltzmann constant, and $T$ the temperature [14]. The susceptibility of the colloidal particles is $\chi_{\text {eff }}=7.5 \times 10^{-11} \mathrm{~A} \mathrm{~m}^{2} / \mathrm{T}$. In the experiment, an external magnetic field $B=0.24 \mathrm{mT}$ is applied, corresponding to $\Gamma \approx 72$.

The experimental system is tilted, and gravitationally induced transport between the two reservoirs has to take place inside the channel. Before starting the experiments, the system is set up completely horizontal. We use configurations where the particles are either all confined in one reservoir or equally distributed along the channel and in both reservoirs.

The BD simulations are based on an overdamped Langevin equation. This approach neglects hydrodynamic interactions as well as the short-time momentum relaxation of the particles. Both approximations are fully justified in the current experimental context. Typical momentum relaxation times are on the order of $100 \mu \mathrm{s}$ and, therefore, much shorter than the repetition rate of the video microscopy setup $\left(\begin{array}{ll}10 & \mathrm{~s}\end{array}\right)$. The colloidal trajectories $\mathbf{r}_{i}(t)=$ $\left(x_{i}(t), y_{i}(t)\right)(i=1, \ldots, N)$ are approximated by the stochastic position Langevin equations with the friction constant $\xi$

$$
\xi \frac{d \mathbf{r}_{i}(t)}{d t}=-\nabla_{\mathbf{r}_{i}} \sum_{i \neq j} V_{i j}\left(r_{i j}\right)+\mathbf{F}_{i}^{\mathrm{ext}}+\tilde{\mathbf{F}}_{i}(t) .
$$

The right-hand side includes the sum of all forces acting on each particle, namely, the particle interaction, the constant driving force $\mathbf{F}_{i}^{\text {ext }}=m g \sin (\alpha) \hat{\mathbf{x}}$, and the random forces $\tilde{\mathbf{F}}_{i}(t)$. The latter describe the collisions of the solvent mole- cules with the $i$ th colloidal particle and in the simulation are given by random numbers with zero mean $\left\langle\tilde{\mathbf{F}}_{i}(t)\right\rangle=0$ and variance $\left\langle\tilde{F}_{i \alpha}(t) \tilde{F}_{i \beta}(0)\right\rangle=2 k_{B} T \xi \delta(t) \delta_{i j} \delta_{\alpha \beta}$. The subscripts $\alpha$ and $\beta$ denote the Cartesian components. These position Langevin equations are integrated forward in time in a Brownian dynamics simulation using a finite time step $\Delta t$ and the technique of Ermak [15]. Particles are confined to the channel by ideal elastic hard walls in the $y$ direction and at $x=0$ (channel entrance). The channel end is realized as an open boundary. To keep the overall number density in the channel fixed, every time a particle leaves the end of the channel, a new particle is inserted at a random position (avoiding particle overlaps) within the first $10 \%$ of the channel, acting as a reservoir.

Starting from a random particle distribution within the channel, we first calculate an equilibrium configuration $\left(\mathbf{F}_{i}^{\text {ext }}=0\right)$ of a closed channel with ideal hard walls. Afterwards, we apply the external driving force and allow the system to reorganize for $10^{6}$ time steps, before we evaluate the configurations. The time step $\Delta t=$ $7.5 \times 10^{-5} t_{B}$ is used, with $t_{B}=\xi \sigma^{2} / k_{B} T$ being the time necessary for a particle in equilibrium to diffuse its own diameter $\sigma$. We choose $\xi=3 \pi \eta \sigma$, with $\eta$ denoting the shear viscosity of the water. The simulations are done with 2000-4500 particles, for a channel geometry of $L_{x}=$ $800 \sigma$ and $L_{y}=(9-12) \sigma, \quad$ and $\quad \chi_{\text {eff,sim }}=3 \times$ $10^{-11} \mathrm{~A} \mathrm{~m}^{2} / \mathrm{T}$. Thus, external magnetic fields $B=$ $0.5-4.0 \mathrm{mT}$ correspond to $\Gamma \approx 80-5500$.

To study the robustness of the formation of the density gradient and its connection to the layer reduction in our system of gravitationally driven particles, we performed simulations for different inclinations $\alpha=0.0^{\circ}-10.0^{\circ}$, keeping the overall particle density fixed at $\rho=$ $0.425 \sigma^{-2}$. The simulations show a linear density gradient, which is maximal for $\alpha=0^{\circ}$ [Fig. 1(b)]. Even at $\alpha=0^{\circ}$, a pressure difference between both channel ends occurs for the boundary conditions used, and a small particle flow is induced. For inclinations $\alpha>1.0^{\circ}$, the density gradient

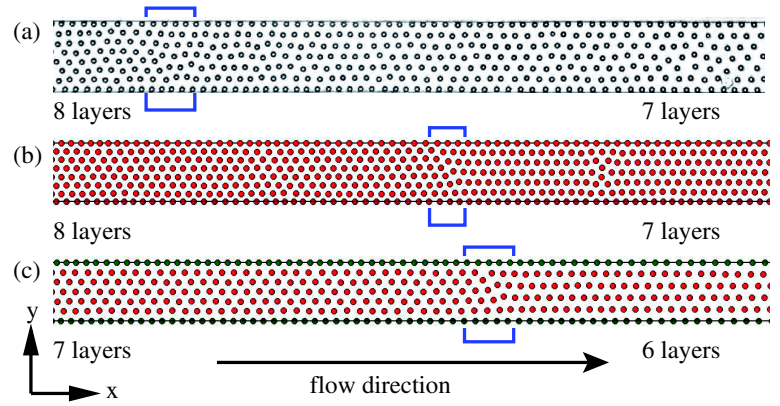

FIG. 2 (color online). (a) Video microscopy snapshot of colloidal particles moving along the lithographically defined channel $(692 \times 60 \mu \mathrm{m}, \Gamma \approx 72)$. (b) Simulation snapshots for a channel with ideal hard walls $(573.3 \times 45 \mu \mathrm{m}, \Gamma=640.5)$; (c) the same as in (b) with the particles at the walls kept fixed $(573.3 \times 45 \mu \mathrm{m}, \Gamma=5026)$. The rectangles mark the region of the layer reduction. 
becomes nearly zero. For these inclinations, the driving force dominates, and we find plug flow of the particles without layer reduction. A decrease of the inclination (driving force) gives rise to an increase of the density gradient. Under nonplug flow conditions, we find a selfinduced rearrangement of the particles to a nearly hexagonal lattice and the occurrence of layer reductions. In the following, we present experimental and simulation results for the inclinations $\alpha_{\exp }=0.6^{\circ}$ and $\alpha_{\text {sim }}=0.2^{\circ}$, where we are in a gravitationally driven nonequilibrium situation but not yet in the regime of plug flow. These inclinations result in an average drift velocity $v_{\text {drift }} \approx 0.035 \mu \mathrm{m} / \mathrm{s}$.

A typical snapshot from the experiment of the particles moving along the channel is shown in Fig. 2(a). Similar snapshots we get from simulations with comoving [Fig. 2(b)] and fixed boundary particles [Fig. 2(c)]. In most regions of the channel, the particles arrange themselves in a nearly hexagonal order. This behavior is due to the strength of the particle interactions caused by the external magnetic field (high $\Gamma$ values), which leads to hexagonal ordering in unbounded systems as well. The formation of this order naturally gives rise to the formation of layers in the motion of the particles along the channel. A similar layering phenomenon has been observed in channels under equilibrium conditions [16]. Additionally to this, we observe, both in experiment and in simulation, a decrease of the number of layers in the direction of motion. In Fig. 2, the regions of layer reduction have been marked. The position of this layer reduction zone does not move together with the particles. We therefore do not observe plug flow of a crystal but rather a dynamic behavior of particles moving in layers and adapting to the external potential.

The reduction of the number of layers originates from a density gradient along the channel. The local particle density inside the channel is shown in Figs. 3(a) and 3(b) together with the particle separations in the $x$ and $y$ directions. In the experiment [Fig. 3(a)], the density decreases monotonically along the direction of the motion of the particles by about $20 \%$. The average density in the channel shows fluctuations on the order of $10 \%$ as a function of time. The total increase in density, however, is less than 3\% during the total time of the experiment. We therefore argue that the density gradient is formed in a quasistatic situation. This argument is confirmed by results of BD simulations [Fig. 3(b)], where the corresponding decrease of the particle density is observed.

The particle separations of neighboring particles in the $x$ and $y$ directions are used to calculate the local lattice constant $d$. Because of the density gradient along the channel, the ordered structure is not in its equilibrium configuration at all points along the channel. Thus, the local lattice constant $d_{x}$, calculated from the particle separations in the $x$ direction, can deviate from the local lattice constant $d_{y}$, calculated from the particle separations in the $y$ direction. At the left end of the channel, $d_{x}$ is larger than $d_{y}$, indicating that the ordered structure is stretched along the $x$ axis. At the point of the layer reduction, the system changes back to a situation where $d_{x}$ is smaller than $d_{y}$. This is achieved by decreasing $d_{x}$ and increasing $d_{y}$ by about $20 \%$ simultaneously. These changes of separations compensate each other and result in a continuous change in the local density at the position of the layer reduction. The behavior of the system shows that the stretching of the ordered structure before the layer reduction causes an instability towards decreasing the number of layers. This decrease compresses the system along the $x$ direction but apparently lowers the total energy of the system.

This scenario can be qualitatively confirmed by the following rough estimation: Starting from an ideal triangular configuration with a given number of layers $\left(n_{l}\right)$ in a channel of fixed width, we calculated the potential energy per particle for different particle densities by just scaling the channel length of the static configuration. Plots of these energies per particle for different values of $n_{l}$ as a function of the particle density are shown in Fig. 3(c). They show
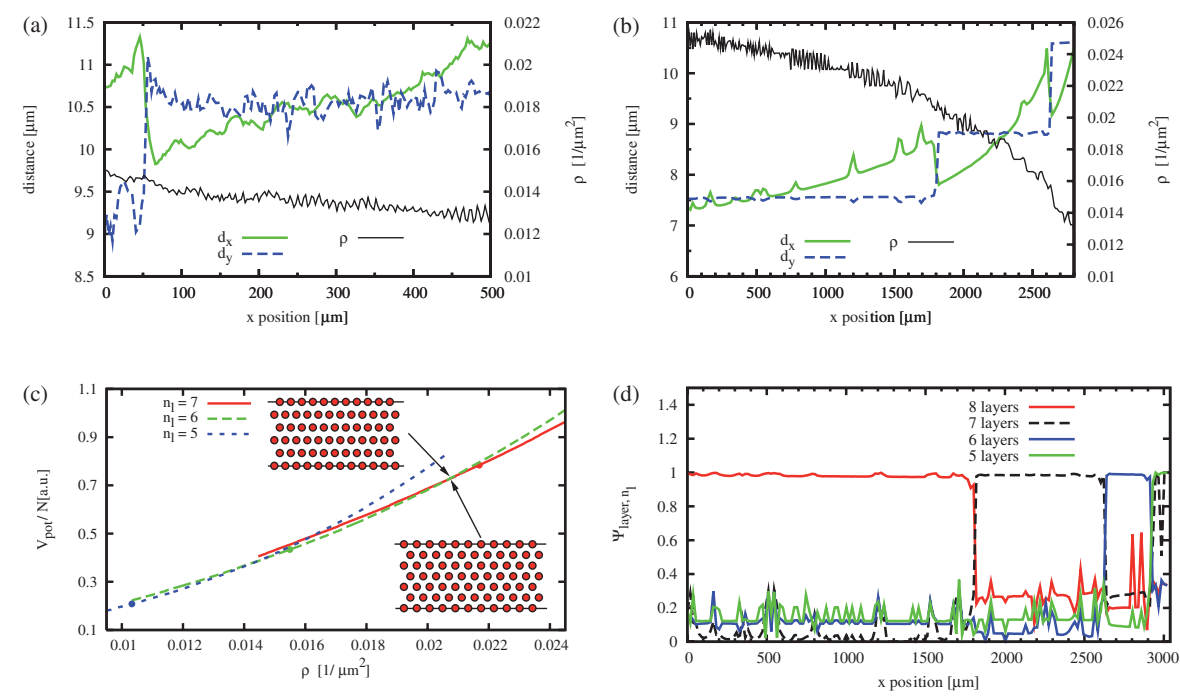

FIG. 3 (color online). Local lattice constants $d_{x}$ and $d_{y}$ and local particle density (a) in the experiment and (b) in the BD simulation. The results are obtained for the systems in Figs. 2(a) and 2(b), respectively. (c) Potential energies per particle of different layer configurations as a function of the particle density. The dots mark the perfect triangular lattices for 5, 6, and 7 layers. Also shown are parts of the configurations with 7 and 6 layers at the intersection point. (d) Plots of the layer order parameter for the configuration snapshot in Fig. 2(b). Multiple points of layer reductions can be identified. For smaller overall densities, we found reductions down to just two layers in simulations. 


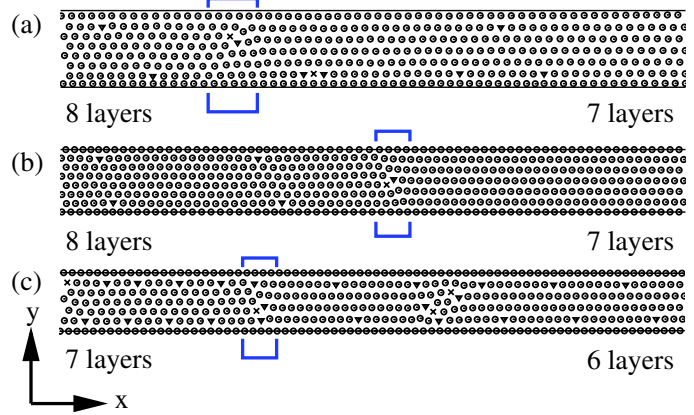

FIG. 4 (color online). Snapshots of defect configurations obtained from a Delaunay triangulation of the particles moving in the channel. The particles are coded according to the number of nearest neighbors they have. Open circles mark the bulk particles with 6 nearest neighbors and the edge particles; $\times$ corresponds to fivefold symmetry and $\nabla$ to sevenfold symmetry. (a) Experiment: In order to minimize the effects of fluctuations on a short time scale, 50 images have been averaged. (b) BD simulation for a channel with parallel walls. (c) Equilibrium BD simulation for a channel with nonparallel walls $\left(\Delta L_{y}=1 \sigma\right.$ between both channel ends, $\Gamma=153.9$, and the channel has 10 times the length of the excerpt shown). In addition to the defect at the layer reduction, defects can be seen close to the walls, because the particle density of the layers at the wall is higher than for "bulk" particles $[16,17]$.

clear intersection points, indicating that for a stretched configuration with $n_{l}$ layers in the $x$ direction it can become energetically more favorable to switch to a compressed configuration with $\left(n_{l}-1\right)$ layers. Also, equilibrium BD simulations $\left(\mathbf{F}_{i}^{\text {ext }}=0\right)$ of closed channels with nonparallel walls result in a density gradient with decreasing channel width and show layer reductions. A snapshot is shown in Fig. 4(c).

The point of layer reduction can be well localized by an appropriate local order parameter. We therefore divide the channel of width $L_{y}$ into several bins in the $x$ direction, each containing $n_{\text {bin }}$ particles, and evaluate for a different number of layers $\left(n_{l}\right)$ the layer order parameter $\Psi_{\text {layer, } n_{l}}=$ $\left|\left(1 / n_{\mathrm{bin}}\right) \sum_{j=1}^{n_{\mathrm{bin}}} e^{i\left(2 \pi\left(n_{l}-1\right) / L_{y}\right) y_{j}}\right|$, which is unity for $n_{l}$ particles distributed equidistantly across the channel width starting at $y=0$. As can be seen in Fig. 3(d), the layer order parameter exhibits a clear discontinuity at the position of the layer reduction. The local orientational order parameter $\Psi_{6}$, which is often used for 2D systems [14], is not so significant for this system, as it is very sensitive to any perturbation of the sixfold symmetry.

In Fig. 4, snapshots of nonequilibrium defect configurations are shown. They reveal that the system is nearly triangular left and right of the point of layer reduction. The change is marked by a single defect only. The number of layers is reduced one by one; reductions of two or more layers have not been observed in experiment or in simulation. Naturally, this reduction produces a defect at the point of the transition. Since the position of the layer reduction is determined mainly by the density gradient, its location remains stable with time on average. A more detailed analysis reveals, however, that the transition point oscillates back and forth around this average position. At the transition, the driven particles in the bulk layers have to change the layer, causing the transition to move a little bit in the direction of the flow. A particle changing into the edge layer can neutralize the defect of the transition locally. This causes a reconfiguration of the ordered structure, which in turn gives rise to repositioning of the layer reduction zone back to a region of higher density.

To summarize, we have shown the formations of layers and the occurrence of a layer reduction in a 2D system of superparamagnetic colloids both experimentally and by Brownian dynamics simulations. The layer formation is induced by the repulsive particle interactions and the confining potential of the channel. Because of those two factors, an ordered structure forms in the channel. This structure is stretched along the direction of the motion of the particles as a response to the boundary conditions at both ends of the channel and the particle interactions. Because of this stretching, a layer reduction occurs in the channel, at which the ordered structure gets locally distorted. After the transition, the ordered structure is compressed in the direction of motion.

We gratefully acknowledge the support of the SFB 513, the SFB TR6, and the NIC, HLRS, and SSC.

*Electronic address: Artur.Erbe@uni-konstanz.de

[1] P. Schall et al., Science 305, 1944 (2004).

[2] R. Roth and D. Gillespie, Phys. Rev. Lett. 95, 247801 (2005).

[3] R. Bubeck, C. Bechinger, S. Neser, and P. Leiderer, Phys. Rev. Lett. 82, 3364 (1999).

[4] K. Zahn, A. Wille, G. Maret, S. Sengupta, and P. Nielaba, Phys. Rev. Lett. 90, 155506 (2003).

[5] B. Schmittmann and R. K. P. Zia, Phase Transitions and Critical Phenomena (Academic, New York, 1995), Vol. 17.

[6] B. v. Wees et al., Phys. Rev. Lett. 60, 848 (1988).

[7] D. Wharam et al., J. Phys. C 21, L209 (1988).

[8] E. Scheer et al., Nature (London) 394, 154 (1998).

[9] M. Dreher, F. Pauly, J. Heurich, J. Cuevas, E. Scheer, and P. Nielaba, Phys. Rev. B 72, 075435 (2005).

[10] P. Glasson et al., Phys. Rev. Lett. 87, 176802 (2001).

[11] G. Piacente and F. Peeters, Phys. Rev. B 72, 205208 (2005).

[12] R. Haghgooie and P. Doyle, Phys. Rev. E 70, 061408 (2004).

[13] A. Ricci, P. Nielaba, S. Sengupta, and K. Binder, Phys. Rev. E 74, 010404(R) (2006).

[14] K. Zahn, R. Lenke, and G. Maret, Phys. Rev. Lett. 82, 2721 (1999).

[15] D. L. Ermak, J. Chem. Phys. 62, 4189 (1975).

[16] R. Haghgooie, C. Li, and P. Doyle, Langmuir 22, 3601 (2006).

[17] R. Haghgooie and P. Doyle, Phys. Rev. E 72, 011405 (2005). 